\title{
Multi-Physics Modeling of RF and Microwave High-Power Transistors
}

\author{
Peter H. Aaen, Senior Member, IEEE, John Wood, Fellow, IEEE, Daren Bridges, Member, IEEE, \\ Lei Zhang, Member, IEEE, Eric Johnson, Member, IEEE, Jaime A. Plá, Member, IEEE, Travis Barbieri, \\ Christopher M. Snowden, Fellow, IEEE, John P. Everett, and Michael J. Kearney
}

\begin{abstract}
In this paper, we present a multi-physics approach for the simulation of high-power RF and microwave transistors, in which electromagnetic, thermal, and nonlinear transistor models are linked together within a harmonic-balance circuit simulator. This approach is used to analyze a laterally diffused metal-oxide semiconductor (LDMOS) transistor that has a total gate width of $102 \mathrm{~mm}$ and operates at $2.14 \mathrm{GHz}$. The transistor die is placed in a metal-ceramic package, with bondwire arrays connecting the die to the package leads. The effects of three different gate bondpad layouts on the transistor efficiency are studied. Through plots of the spatial distributions of the drain efficiency and the time-domain currents and voltages across the die, we reveal for the first time unique interactions between the electromagnetic effects of the layout and the microwave behaviour of the large-die LDMOS power field-effect transistor (FET).
\end{abstract}

Index Terms-Global modeling, electrothermal, laterallydiffused metal-oxide-semiconductor (LDMOS) transistor, power field-effect transistor (FET).

\section{INTRODUCTION}

$\mathbf{P}$ OWER transistors for wireless infrastructure applications have become increasingly complex over the past two decades, from a single die mounted in the package to sophisticated multi-chip modules, as shown in Fig. 1. Modern high-power devices have total gate widths of several hundred millimeters, achieved by connecting many gate fingers in parallel. The packaged transistors are further complicated by in-package matching networks, comprising several bondwire arrays connecting metal-oxide semiconductor (MOS) capacitors, and other passive circuit elements, to create the matching networks. The 200-W transistor in Fig. 1 contains almost 250 individual bondwires.

Market demands for increased power and efficiency show no sign of abating, and more circuit functions are expected to be integrated within the package. The complexity of the design and construction of high-power microwave transistors continues to increase.

In spite of this increasing complexity of the power transistor device, the compact models that are provided by the device manufacturer are often simplified, reducing the arrays of

P. H. Aaen, D. Bridges, L. Zhang E. Johnson, J. Plá, and T. Barbieri are with the RF Division, Freescale Semiconductor Inc., Tempe, AZ, 85284, USA (e-mail: Peter.Aaen@freescale.com)

J. Wood was with Freescale Semiconductor Inc. and is now with Maxim Integrated Products Inc., San Diego, CA, 92121, USA.

C. M. Snowden, J. P. Everrett, and M. J. Kearney are with the University of Surrey, Guildford, Surrey, GU2 7XH, UK.

This paper is an expanded paper from the IEEE MTT-S Int. Microwave Symposium held on June 17-22, 2012 in Montreal, Canada.

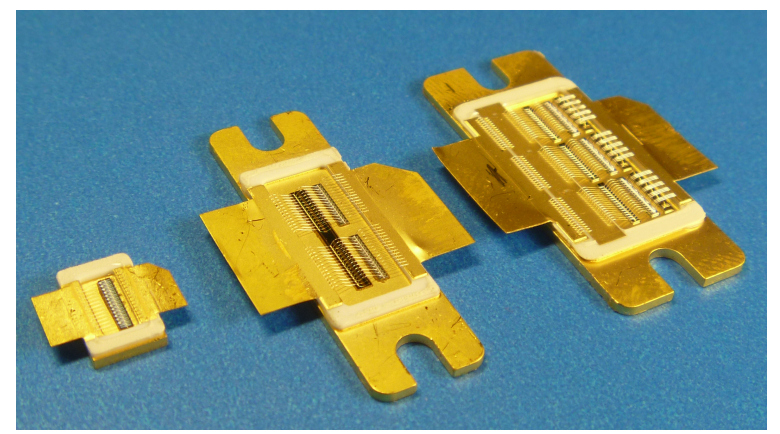

Fig. 1. Photograph showing the increasing complexity of high-power transistors used in wireless infrastructure products over the past 15 years. The internals of a $45-\mathrm{W} 1-\mathrm{GHz}$ and a $60-\mathrm{W} 2-\mathrm{GHz}$ transistor (on the lefthand side and center) are compared with a modern 200-W 2-GHz packaged transistor (on the right-hand side). Note the large increase in the number of components and complexity in the matching networks.

bond wires and capacitors to a few lumped components, and ignoring distributed effects. This has the benefit of reducing the model to only a few essential ports, simplifying the model extraction and speeding up the circuit simulation [1]-[5]. Apart from a few notable exceptions [1], [6]-[13], the internal operation of the packaged transistor is described only coarsely, and the voltages and currents are only available at a few nodes of interest, effectively obscuring the detailed internal operation of the packaged device. Because of this low number of internal nodes, we are limited in our ability to explain poor performance scaling [7], [14] as well as observed temperature distributions that are dependent on frequency, power, and load termination [6].

Fully coupled multi-physics approaches to modeling transistors, where the governing equations are all solved simultaneously, have been developed by a number of researchers [15][17]. While undoubtedly valuable for physically small, highfrequency transistors, the physical scale and consequent computational expense prohibit these approaches for high-power microwave transistors. To overcome the computational expense, 'weakly-coupled' approaches have been defined where electromagnetic simulation results (through S-parameters), thermal models (through a thermal impedance matrix), and nonlinear electrothermal transistor models are coupled in the netlist of a circuit simulator. This provides a comprehensive description of the transistor for computationally efficient simulation [6]-[8], [12], [18].

Weakly-coupled approaches for distributed multi-physics modeling are not without their own expense. For example, 
schematic representations can become unwieldy, model evaluation can suffer from long simulation time, and data processing can be intense. With the availability of cluster computing, it is possible to simulate circuits containing hundreds of nonlinear transistors, connected to fully distributed matching networks and their interconnections to distributed thermal models. Using this approach, the voltages and currents at all nodes of interest within the packaged device are available, and they can be interrogated to develop a better understanding of its internal operation.

In this paper, we present a multi-physics methodology that combines electromagnetic and thermal simulations with nonlinear electrothermal transistor models in harmonic-balance circuit simulations. In Section II, we present the methods by which we develop the constituent models. We also introduce an experiment where the simulation methodology and models are used to explain performance changes exhibited for three different packaged 102-mm LDMOS transistors where modifications have been made to the layout of the gate bondpad metallization. In Section III, we present a comparison of measured and simulated temperature distributions and largesignal performance. In Section IV and V we present for the first time the visualization of the spatially distributed drain efficiency and time-domain voltages and currents across an electrically large die. As will be shown, these visualization methods provide unique insights into the operation of highpower microwave packaged transistors, which can be very beneficial in the development of future designs. Finally, we conclude the paper in Section VI.

\section{Model Development}

The packaged LDMOS transistor we examined is illustrated in Fig. 2. This device has 204 individual gates, each having a $500 \mu \mathrm{m}$ unit gate width. The individual transistors are fed in pairs, with two gates attached to the metallization that connects to the gate bondpad. The individual drains are connected together in a similar way. On the outer edges of the die only one individual transistor gate is connected to the gate bondpad. Of the 204 gates, six pairs are not connected to the gate and drain manifolds. These non-functioning 'dead' fingers were relics of a separate experiment where the output power of the transistor could be tailored with only minor metallization changes. As will be seen, the dead fingers contributed significant detail to the temperature distributions along the center of the die.

We used this strategy of defining the power transistor in terms of its constituent electromagnetic, thermal, and nonlinear electronic component parts, to investigate the relationships between the internal behaviors and the terminal performance of the transistor. We have called this strategy a multi-physics modeling methodology, as it couples directly the important physical environments that affect the operation of the device.

We apply the multi-physics modeling methodology to study the effects of changing only the gate bond-pad structure on the device performance. The bondpad width has values of $w=0,80$, and $160 \mu \mathrm{m}$, as illustrated in Fig. 3. For $w=$ $160 \mu \mathrm{m}$, the bondpad has discrete areas for the bondwires to connect, whereas the $w=0 \mu \mathrm{m}$ bondpad is uniformly

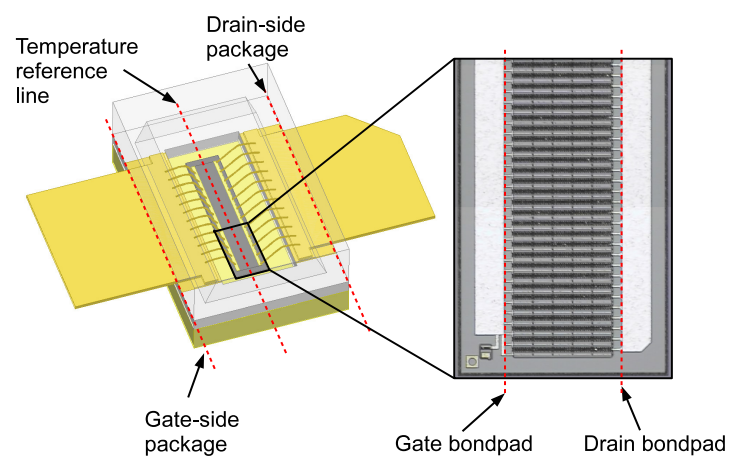

Fig. 2. An illustration of the packaged transistor with a transparent ceramic lid revealing the gate and drain bondwire arrays and their connection to the LDMOS die. All reference planes of interest are indicated by dashed lines for later reference.

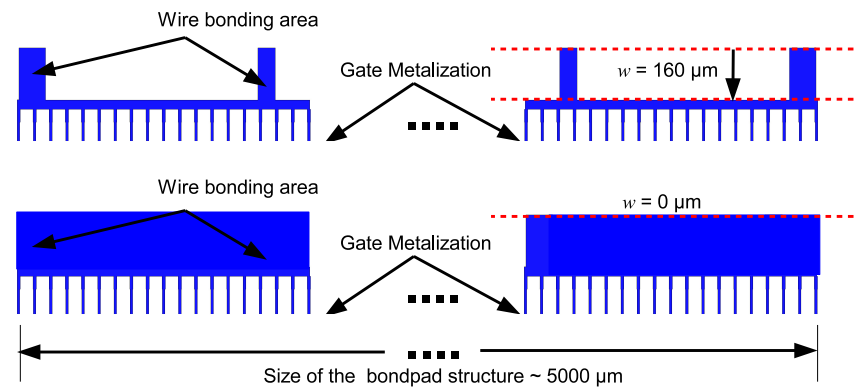

Fig. 3. An illustration of two gate bondpads with the port connections for the bondwires and connections to the gates of the transistors. The metallization width, $w$ takes on the values $0,80,160 \mu \mathrm{m}$.

wide. A packaged device for each value of $w$ was measured in a loadpull test bench over a range of input power from 23 to $37 \mathrm{dBm}$ at $2.14 \mathrm{GHz}$. Harmonic-balance simulations were performed over a range of source and load impedances to simulate load pull of the packaged transistors.

In the following sub-sections we present the development of each constituent component of the packaged transistor and explain in detail how the multi-physics model is constructed.

\section{A. Transistor Model Development}

Our comprehensive model incorporates a measurementbased nonlinear electrothermal transistor model [19], which was extracted from a $5.0-\mathrm{mm}$ on-wafer transistor. A dense pattern of pulsed I-V and S-parameter measurements are taken over the gate-drain voltage space of the transistor, bounded by the maximum drain current, breakdown voltage, and the maximum allowable power dissipation. The manifold structure and the extrinsic network are de-embedded to obtain Sparameter data at the intrinsic model reference planes [20]. After converting to Y-parameters, the LDMOS transistor model current and charge state functions $\left(I_{d}, Q_{g}\right.$, and $\left.Q_{d}\right)$ can then be obtained by integration of the small-signal voltage-dependent parameters [14]. This integral formulation for determining the charges ensures a conservative charge formulation, essential for accurate prediction of low-level phase nonlinearity, and for convergence in time-domain simulations [14], [21]. 
In our model extraction process we obtain charge data indexed by the intrinsic gate and drain voltages. These data are then approximated using artificial neural networks (ANNs), resulting in smooth and infinitely differentiable two-dimensional charge functions that are used directly in the model.

For the drain current, we use the analytical expression (1) from [22] as this expression has been shown to fit accurately the current data in the near-threshold region. This is typically where the LDMOS power transistor is biased for power amplifier applications:

$$
I_{\mathrm{d}}=\frac{\beta V_{\mathrm{gst}}^{2}}{1+V_{\mathrm{gst}}^{p} / V L} \tanh \left(\frac{\alpha V_{\mathrm{ds}}}{V_{\mathrm{gst}}^{p s a t}}\right)\left(1+\lambda V_{\mathrm{ds}}\right)
$$

where $\beta$ and $V L$ are parameters that control the slope in the quadratic region and the transition to the linear region. The parameter $p$ allows the slope of the transconductance to be modified in the linear region, $V_{g s t}$ is the gate control function, and $\lambda$ and $\alpha$ are additional fitting parameters. The current in the sub-threshold region and between the quadratic region is modeled with $\beta V_{g s t}^{2}$ where

$$
V_{g s t}=V S T \ln \left(1+e^{\left(V_{g s}-V_{t}\right) / V S T}\right)
$$

and $V_{t}$ is the threshold voltage, $V S T$ controls the abruptness of the 'turn-on' characteristic, $V_{g s}$ is the gate-to-source voltage, and $V_{d s}$ is the drain-to-source voltage.

The effects of self-heating on the drain current are incorporated using a self-consistent electro-thermal model [23]. A straight-forward method of including the thermal effects on the output current is to use a de-rating function on the drain current expression [14], [24], and it can be expressed as:

$$
I_{\mathrm{d}}=\frac{I_{\mathrm{d} 0}}{1+\frac{\left(T-T_{0}\right)}{T_{0}}}=\frac{I_{\mathrm{d} 0}}{1+\frac{R_{\mathrm{th}} P_{\mathrm{avg}}}{T_{0}}}
$$

where $T$ is the temperature, $I_{d 0}$ is the drain current measured at a reference temperature $T_{0}, R_{t h}$ is the thermal resistance, $P_{a v g}$ is the average of the dissipated power,

$$
P_{\text {diss }}(t)=\frac{\left(T(t)-T_{0}\right)}{R_{\mathrm{th}}}+C_{\mathrm{th}} \frac{d}{d t}\left(T(t)-T_{0}\right)
$$

and $C_{t h}$ is the thermal capacitance.

During a harmonic-balance simulation, the power dissipated as computed by the transistor model is passed to the thermal model. The temperature rise is then computed and it is then passed back to the transistor model. This process continues until convergence is reached.

Once the model has been extracted based upon the characterization data obtained from the 5.0-mm transistor, the model is scaled down to the $500 \mu m$ unit-gate width. One such model is used to represent each gate finger of the transistor; this is our unitary transistor model.

\section{B. Thermal Model Development}

The thermal model for the entire packaged transistor is obtained through finite-element based simulations using ANSYS ${ }^{\mathrm{TM}}$. In these simulations, the bottom of the package flange is held at a constant temperature. All other surfaces are

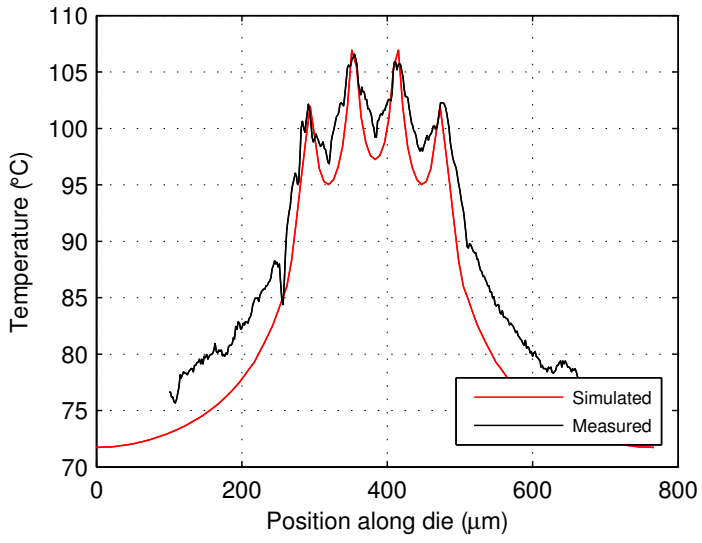

Fig. 4. Measured and simulated temperature across the a 4.8-mm LDMOS transistor [25].

assumed to be adiabatic, and for simplicity we neglected the heat-spreading effects of the metallization on the die, as well as convective and radiative cooling.

As reported in [25], we demonstrate the accuracy of our finite-element simulations by comparison with measurements of an LDMOS transistor biased under DC conditions to obtain $4.8 \mathrm{~W}$ of dissipated power. In this example, the transistor had a total gate width of $4.8 \mathrm{~mm}, 600 \mu \mathrm{m}$ unit-gate width, $30 \mu \mathrm{m}$ source-drain pitch, is built on an approximately $80 \mu \mathrm{m}$ thick silicon substrate, and is mounted on top of a $730 \mu \mathrm{m}$ thick copper carrier. Simulations of the same device were performed and plots of the temperature profiles across the transistor are shown in Fig. 4. The measurements are performed using a QFI InfraScope II ${ }^{\mathrm{TM}}$ system outfitted with a $15 \times$ magnification lens resulting in a spot size of $1.6 \mu \mathrm{m}$.

Using the finite-element method, we generate the thermal resistance matrix $\left(R_{t h}\right)$ for the packaged transistor by turning on an incident heat-flux at each finger and then examining the temperature over the whole die, and measuring the temperature at all other fingers. We proceed by exciting each finger in order, until the full thermal resistance matrix is obtained [6]. The temperature for a given finger is defined by averaging the temperature by integration over the finger area. Since the material properties change with temperature, we are careful to perform these single source simulations at temperatures close to those expected in the solution [26]. The thermal resistance matrix for the devices under study has 204 ports, each port being connected to the thermal node of the electro-thermal transistor model, and is written as a Touchstone file for easy inclusion in the circuit simulator.

\section{Electromagnetic Simulations}

The electromagnetic environment seen by the device plays an important role in its terminal behavior, as will be shown later. This electromagnetic environment includes the on-die metallization of the transistor - the bond pads, the gate and drain metallization structures - and the package and bondwires that connect the device to the outside world. The metallization structure is simulated using a planar electromagnetic simulator, where the substrate definition has been carefully 
determined beforehand using transmission line measurements made over the passive and active regions of the transistor [20]. This simulation has ports where the bond-wires connect to the bonding pads and ports where the gates and drains of each unitary transistor connect to the bonding pads. The resulting S-parameter model has over 200 ports [13]. The package and bond-wires were simulated using a three-dimensional finite element electromagnetic simulation, yielding a multi-port $\mathrm{S}$ parameter model [27]. This model has two ports representing the connections of the complete device to the external circuit, and ports at the ends of each of the gate and drain bondwires. These two multi-port S-parameter models are connected in cascade and enable us to include the model of the electromagnetic environment in a simple manner.

\section{Model Integration - Enabling Multi-physics}

Our multi-physics model couples together models of the electromagnetic environment of the transistor, that is the electromagnetic simulations of the package and die metallization, a thermal model of the die, and nonlinear electro-thermal models representing each gate finger in the large LDMOS FET die. The constituent component models are generated independently as described in the preceding subsections.

Following [6], the gate and drain of each of the 204 unitary transistor models is connected to the appropriate ports of Sparameter matrix representing the bondpad metallizations. The thermal resistance matrix is included through a 204-port (one port for each unitary transistor) impedance matrix. The dissipated power from each unitary transistor model is computed and is provided to the thermal model, which in turn provides the temperature increase resulting from the dissipated power and the thermal coupling with the other unitary transistors. Finally, the model for the 102-mm LDMOS die is connected to the ports of the S-parameter matrix representing the package and bonding wires.

A schematic representation of the connections of the various models is shown in Fig. 5. In a harmonic-balance simulation, each finger is simulated individually, and by monitoring the voltage and currents at each node in the circuit we obtain the temperature distribution and we can compute the performance of each unitary transistor. Simulations of the packaged transistor performance for changes in frequency, impedance terminations, or ambient temperatures are readily made. Modifications to the gate bondpad metallizations are incorporated by changing only the appropriate S-parameters file.

\section{RESUlts}

\section{A. Measurement Setup}

The high output power capability of the packaged transistor is achieved by connecting the 204 unitary transistors in parallel. As a result the input and output impedances that need to be presented to the transistor for optimal operation are very low. Obtaining repeatable loadpull measurements on transistors with very low impedance using mechanical tuners based on $50-\Omega$ slotted transmission lines is very difficult. To

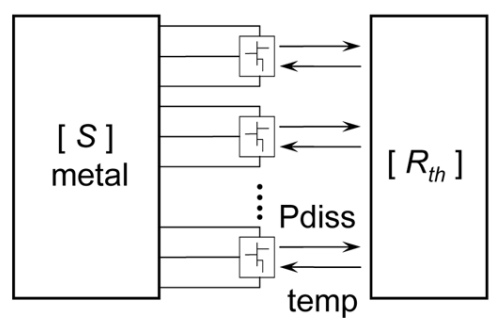

Fig. 5. A diagram showing the interconnection of the various models within the circuit simulator (from [6]).

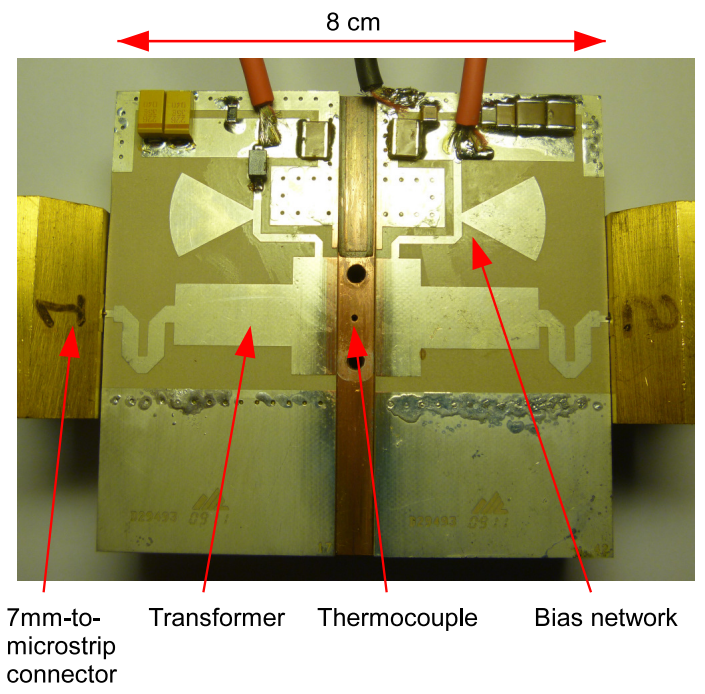

Fig. 6. A photograph of the test-fixture used during loadpull measurements of the packaged transistor. Key elements of the fixture are indicated.

overcome the limitations posed by mechanical tuners, prematching networks are used to transform the tuner impedances to lower values [28]-[30].

In our test-fixture a 10:1 transformer, having a $20 \mathrm{~dB}$ return loss or better over the frequency range $1.5-3.5 \mathrm{GHz}$ was placed between the tuner and the packaged transistor. The test-fixture was characterized using thru-line-reflect (TRL) calibrations for loadpull fixtures [29] and the resulting S-parameters were deembedded from the measurements.

A photograph of the test-fixture is shown in Fig. 6, where the microstrip transformers, bias networks, and the $7 \mathrm{~mm}$ to-microstrip connectors are indicated. A thermocouple is mounted through a small hole, approximately $250 \mu \mathrm{m}$ in diameter, directly beneath the package. The thermocouple monitors the temperature at the backside of the package flange during a loadpull measurement. The temperature rise is computed from the difference between the temperature at the top surface, obtained from IR microscopy, and the temperature measured using the thermocouple.

\section{B. Two-Port Results}

The measured and simulated power-added efficiency (PAE) as a function of the input power for the three different gate bondpad layouts $(w=0,80,160 \mu m)$ are shown in Fig. 7. For each measurement and simulation the load is 


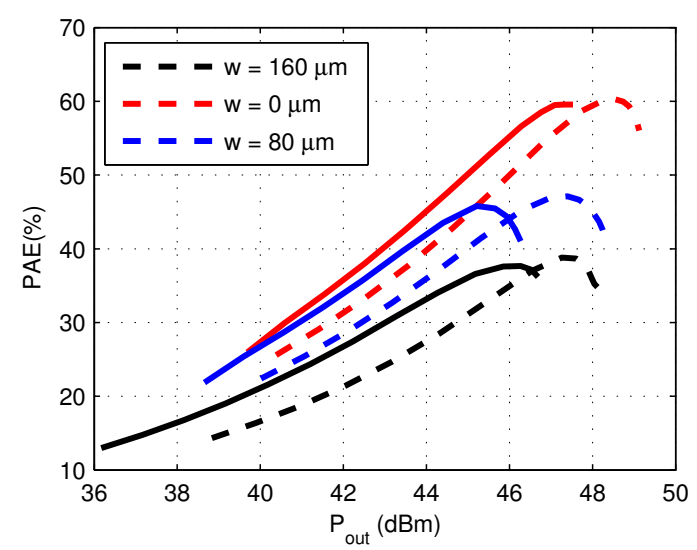

Fig. 7. A comparison of measured vs. simulated power-added efficiency for the three different gate bondpad metallizations. The measured results are indicated by dashed lines and solid lines indicate the simulated results.
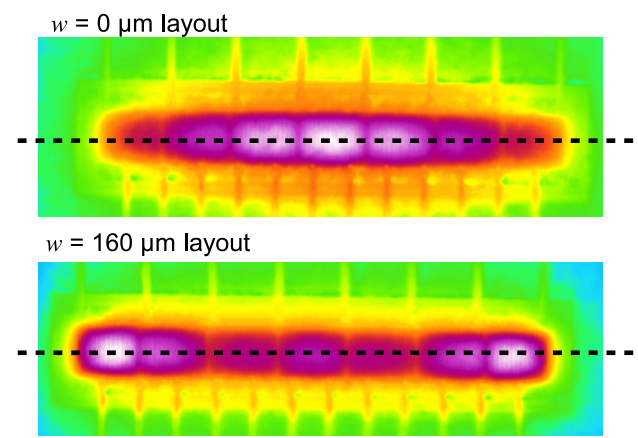

Fig. 8. Infrared images of the die for the bondpads with $w=0 \mu \mathrm{m}$ and $w=160 \mu \mathrm{m}$. The dashed lines indicate the locations of temperature profiles plotted in Figs. 9 and 10

conjugately matched for maximum efficiency and the source is matched when the transistor is operating at $P_{1 d B}$. The uniform bondpad, $w=0 \mu \mathrm{m}$, has approximately $20 \%$ higher poweradded efficiency than the discrete-like bondpad, $w=160 \mu \mathrm{m}$.

The overall shapes of the curves and the maximum efficiency values are well-predicted, although there is a systematic discrepancy between the measured and simulated data. This difference is indicative of a slightly different source match between the measured and simulated results. Although we have used pre-matching transformers, the mechanical tuners are lossy and have finite steps unlike the continuously variable impedances available during a loadpull simulation. The slightly lower peak efficiencies predicted by the models indicate an increased loss present in the model over that of the measured devices. While not shown here, the agreement between other measured and simulated parameters such as gain and output power are broadly similar. The observed differences do not affect the overall predictive performance of this modeling technique in terms of illustrating the important internal behaviors of the device, as will be demonstrated in Section IV.

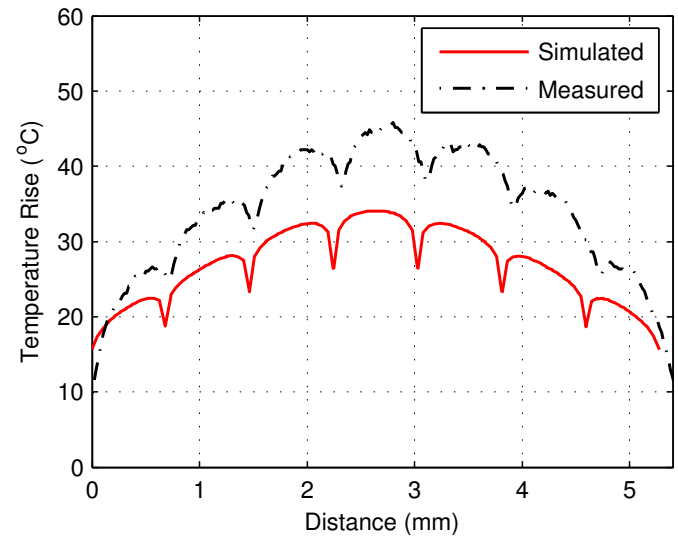

Fig. 9. Measured vs. simulated temperature profiles for the packaged transistor with uniform gate bondpad $w=0 \mu \mathrm{m}$

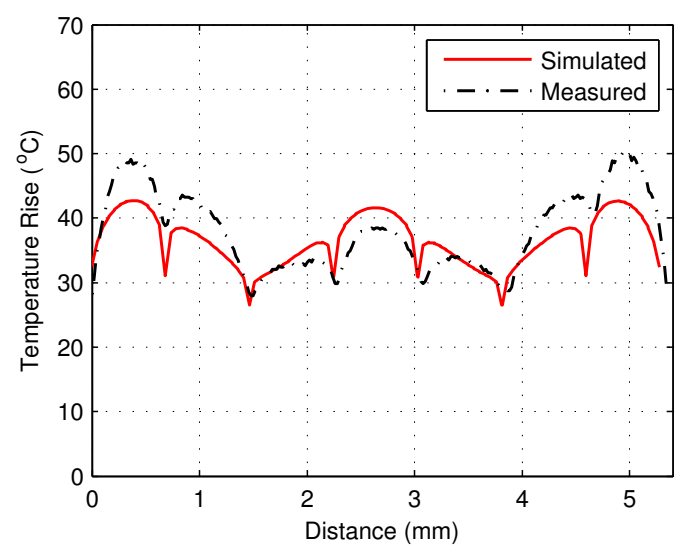

Fig. 10. Measured vs. simulated temperature profiles for the packaged transistor with the discrete gate bondpad $w=160 \mu \mathrm{m}$.

\section{Temperature Distributions}

While our proposed simulation methodology reports voltages and currents at all of the internal nodes, it is very difficult to verify directly the distributed behaviour. Passive and electro-optic field probing methods are an attractive option [31]-[33]. Unfortunately, the construction of the transistor and the closely spaced bondwires between the gate and the drain prevent standard probes from being lowered close enough to the transistor for sufficient resolution of the fields.

To verify the distributed behaviour, we turn to infrared microscopy [34] and compare the measured and simulated temperature rise across the center of the die. The packaged transistors were mounted in a test-fixture and excited at approximately $23 \mathrm{dBm}$ input power. The resulting temperature distributions for two of the manifold cases are shown in Fig. 8. The expected broad 'upside-down U-shape' temperature distribution is seen for the uniform bondpad device [25], but for the device with the discrete bondpads, an interesting triple-peaked distribution is observed.

The simulations are able to reproduce the same behaviour seen in measurements for both manifolds, as shown in Figs. 9 and 10. The dead finger locations can also be identified as low-temperature regions along the transistor die. 


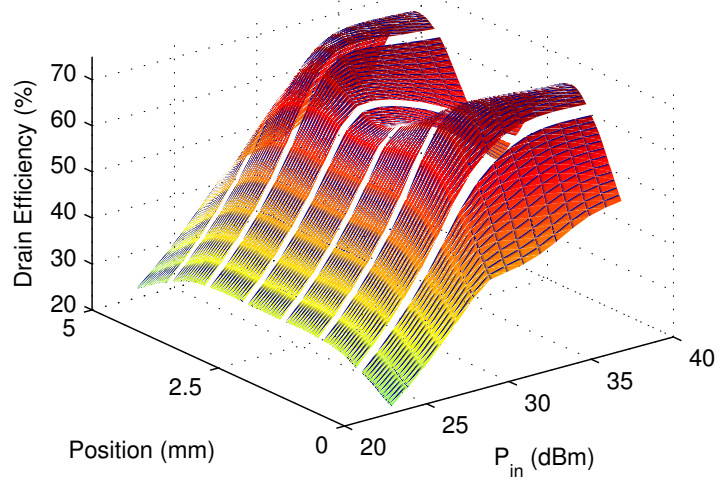

Fig. 11. Spatially distributed drain efficiency for the device with uniform gate bondpad, $w=0 \mu \mathrm{m}$.

The differences between the simulations and measurements are because the IR measurement reports the peak temperature in the middle of each $500 \mu \mathrm{m}$ finger. The simulation uses the thermal resistance matrix that was obtained by averaging the temperature over the unit gate width. The averaging effect of the $1.6 \mu \mathrm{m}$ spot size from the IR camera additionally complicates the comparison. It is important to note that the same thermal model is used to compute the temperature rise in Figs. 9 and 10, where only the gate bondpad layout has been changed.

\section{Spatially Distributed Performance}

In our simulations, the voltages and currents are available for the DC, fundamental, and all the harmonics specified in the circuit simulation, and at every node of interest in the large circuit. Specifically, during the simulation of these packaged transistors (comprising 204 unitary transistors), we collect voltages and currents from the unitary transistors, the edges of the package, the end of the wires, and both sides of the manifolds as illustrated in Fig. 2: this amounts to over a thousand nodes. For a harmonic-balance simulation swept over 15 input powers and including 3 harmonics, this yields a 2 MB text file containing all of the voltages and currents.

With this information, we compute the performance of each unitary transistor and plot the data as a function of transistor position along the width of the bondpad. In Fig. 11, we plot the drain efficiency as a function of the input power and the location of each pair of transistors connected to the drain bondpad. For the device with the uniform gate bondpad, $w$ $=0 \mu \mathrm{m}$, the efficiency exhibits double peaks with a drop in efficiency at the center of the die. For the device with the discrete bondpad, $w=160 \mu \mathrm{m}$, the drain efficiency surface is remarkably different, with the center of the die exhibiting negative efficiency, as shown in Fig. 12. The center of the die is not generating power as expected, but is in fact dissipating power.

Using the voltages and currents from the harmonic-balance simulation, we constructed the time-domain waveforms for each pair of unitary transistors that connect to the drain bondpad. Using these waveforms, we plotted the dynamic

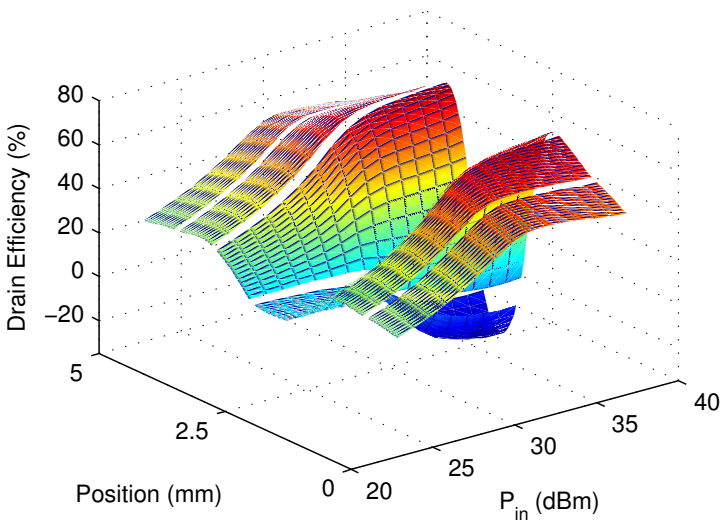

Fig. 12. Spatially distributed drain efficiency for the device with discrete gate bondpad, $w=160 \mu \mathrm{m}$.

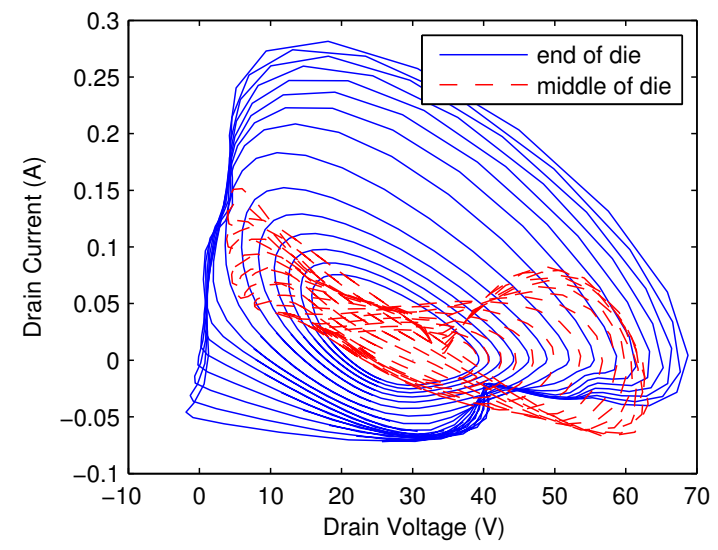

Fig. 13. Dynamic loadlines for the device with the uniform gate bondpad plotted as a function of input power, swept from 23 to $34 \mathrm{dBm}$, in steps of 1 $\mathrm{dBm}$, for the pair of FETs attached to the the edge of the drain bondpad vs. a pair of FETs attached half of the distance down the drain bondpad. Both sets of dynamic loadlines are for the uniform bondpad, $w=0 \mu \mathrm{m}$.

loadlines, over a range of input powers, for a pair of transistors at the end of the drain bondpad and for another pair in the center, as shown in Fig. 13. Although all unitary transistor models are identical, they are operating dramatically different from one another. This is a consequence of the interactive coupling between the transistor, thermal, and electromagnetic models as described in Section II.

In Figs. 14 and 15, we have generated composite plots of the time-domain voltages, currents, and power loss for all unitary transistors. Each plot contains the time-domain waveform for all unitary transistors across the die. By placing each timedomain waveform next to its neighbor, we have in effect created a time-domain surface wave. The wave shows the spatial and temporal distribution of the voltages and currents. By multiplying the voltages and currents at the drains, we can compute the dissipated power per time cycle for each unitary transistor.

We demonstrate the effects of changing the gate bondpad layout by plotting in 3-D the current waveforms for the two limiting cases, $w=0$ and $160 \mu \mathrm{m}$, as shown in Figs. 16 and 17 . We can incorporate the time-dependence and create animations 

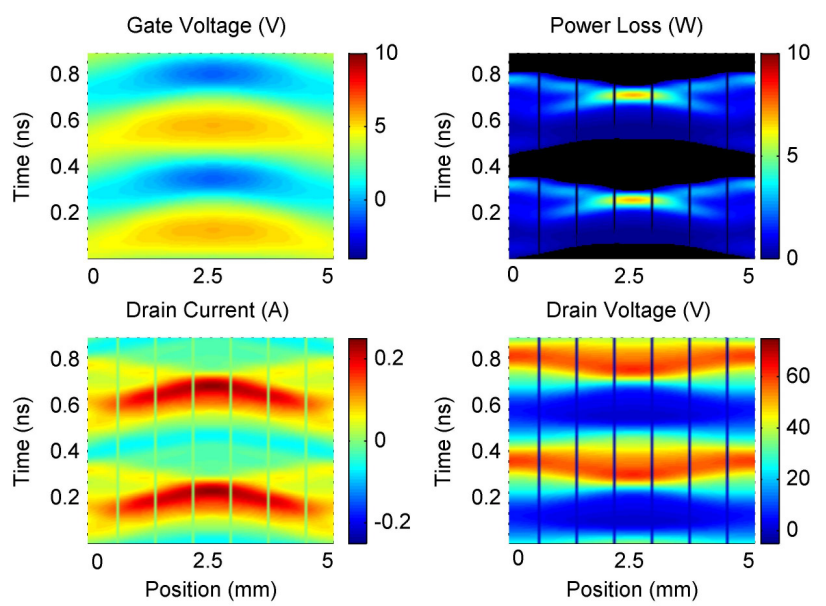

Fig. 14. Time-domain large-signal voltage and current waveforms due to the gate manifold with $w=0 \mu \mathrm{m}$.
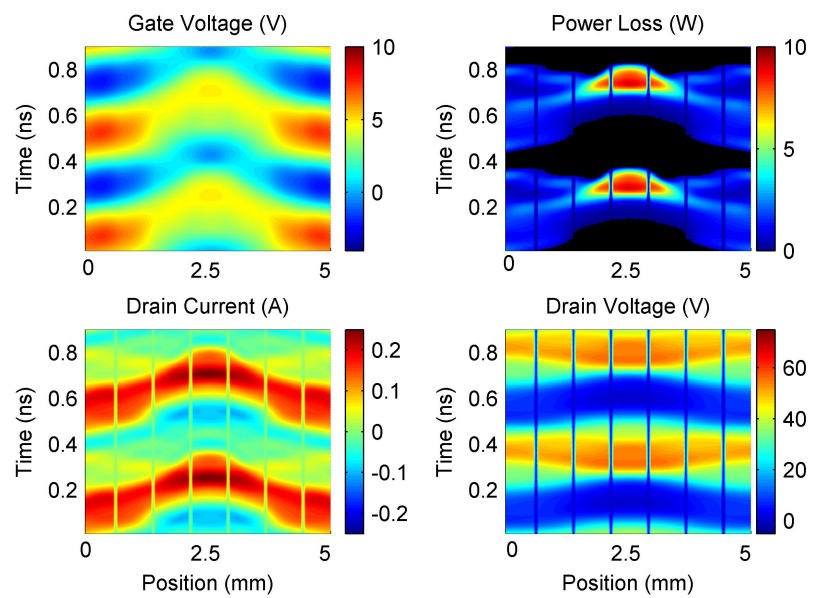

Fig. 15. Time-domain large-signal voltage and current waveforms due to the gate manifold with $w=160 \mu \mathrm{m}$.

of the output current (or any other time-varying parameter). To the best of the authors' knowledge, this is the first time that the visualization of the output current as a function of time and position has been created for the entire die.

\section{Transistor Performance Analysis}

The composite plots that show the gate voltage waveforms in Fig. 14 and 15 are surprisingly very different from one another. The maximum gate voltage for the die with $w=160$ $\mu m$ appears at the center of the die much later than it does at the edges. Since this voltage distribution controls the drain current for each individual transistor, the output current from the transistors in the center of the die is also retarded. The maximum gate voltage for the die with $w=0 \mu \mathrm{m}$ excites practically all unitary transistors simultaneously and the drain current distribution is virtually in-phase across the width of the bondpad. Note that the vertical lines showing no current are the locations of the non-functioning fingers, as described in Section II.

From the drain voltage and drain current surfaces we can compute the dissipated power surface. The plots generated in

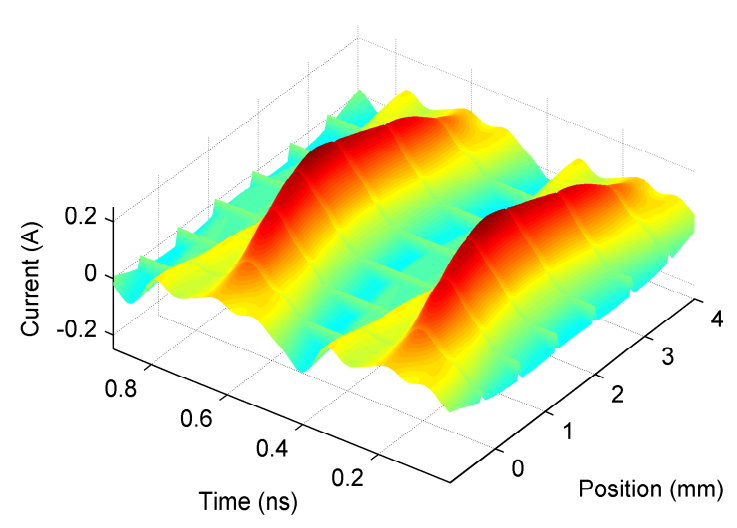

Fig. 16. Visualization of the drain current as a function of time and location within the transistor; PAE $\approx 60 \%$ and $w=0 \mu \mathrm{m}$.

(Animated version is available by clicking here or by opening the file TMTT2012-07-0634_Fig16_animation.avi.)

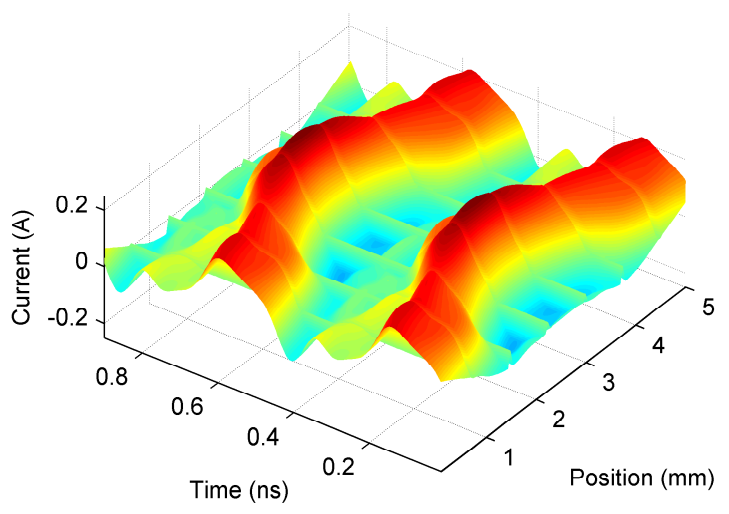

Fig. 17. Visualization of the drain current as a function of time and location within the transistor; $\mathrm{PAE} \approx 40 \%$ and $w=160 \mu \mathrm{m}$.

(Animated version is available by clicking here or by opening the file TMTT2012-07-0634_Fig17_animation.avi.)

Figs 14 and 15, titled 'Power Loss,' were computed by taking the product of the instantaneous drain current and drain voltage waveforms. To show only the loss, the values of positive power generation were set to zero, and these are represented by the black regions. The total area of the black region is dictated by the conduction angle of each unitary transistor and the amount of overlap between the current and voltage waveforms. A transistor exhibiting $100 \%$ efficiency would be represented by an entirely black plot.

Computed power-loss plots for each device show dissipation in the center of the die. This is the reason for the drop in the drain efficiency in the center of the die for the device with the uniform bondpad and for the huge drop in efficiency for the device with the discrete bondpad. In fact, for the device with discrete bondpads, the drain voltage and current surfaces are sufficiently aligned that the center of the transistor is operating as an active resistor. The delayed voltage distribution in the center of the large transistor is a result of the distributed electromagnetic effects from the discrete gate bondpads in combination with the bondwires and package, as noted in [7]. 


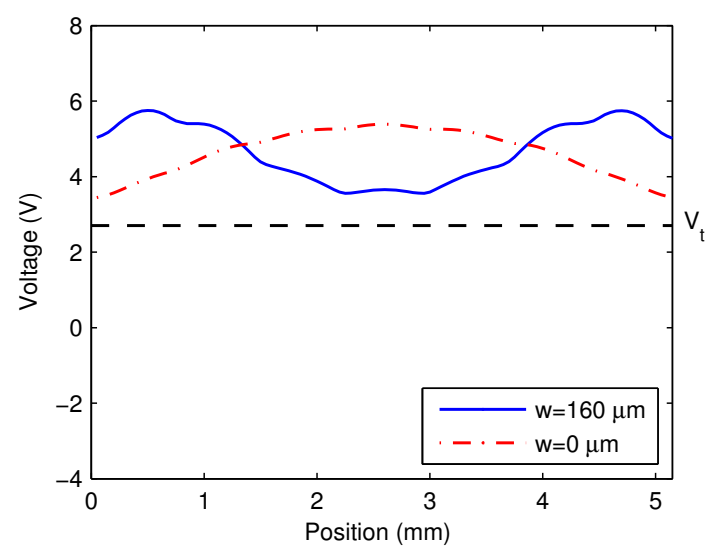

Fig. 18. Voltages on the gates for each pair of transistors along the two gate bondpads. Note that in both cases there are significant voltage variations across the transistor. The threshold voltage of the unitary transistor model is indicated.

(Animated version is available by clicking here or by opening the file TMTT2012-07-0634_Fig18_animation.avi.)

In Fig. 18, plots of the voltages applied to gates of the unitary transistors as a function of position along the gate bondpads are shown. For the device with the discrete bondpad, the applied gate voltage in the center of the die is much lower than at the ends. This plot shows the voltages at a single instant in time; it represents a single horizontal line on the plots of gate voltage as a function of time and position as shown in Figs. 14 and 15. If the data traces in Fig. 18 are animated as a function of time, we can show that for the device with the discrete bondpads, the voltage applied to the unitary transistors in the center of the die is considerably delayed, compared with the voltage applied at the edges.

To investigate the origins for the retarded gate voltage due to the discrete bondpad, we used Sonnet's commercially available em planar electromagnetic simulator and emvu to plot the current distributions. The large-signal currents on the bondpads can be generated once the nonlinear circuit simulation is completed. The voltages obtained from the harmonicbalance simulation, at every node connected to the bondpad, are exported and applied to all of the ports in emvu [35]. The resulting current distribution is the in-situ current seen on the manifold while the packaged transistor is in large-signal operation. In Fig. 19 we plot the current for the fundamental frequency, $2.14 \mathrm{GHz}$ for both manifolds, and each plot is on the same current density scale.

For the packaged transistor with the discrete bondpad, large currents flow in the thin metal connecting the discrete bondpads and along the gates of the unitary transistors as seen in Fig. 19. These transverse currents take time to propagate and the time delay causes the unitary transistors located in the center of the die to conduct later than those at the edges of the die. For the packaged transistor with a uniform gate manifold, minimal transverse currents are observed and there is significantly less voltage variation across the gates of the unitary transistors.

\section{CONCLUSION}

A multi-physics methodology, which combines nonlinear electrothermal transistor models for each individual gate finger with electromagnetic simulations and a thermal model in a nonlinear circuit simulator, has been presented. This methodology was applied to study the effects of different gate bondpad layouts on the operation of a physically large, high-power LDMOS transistors. The shape of gate bondpads was shown to have a large influence on the transistor performance. In the worst case, individual transistors at the center of the die were dissipating rather than generating power. Using distributed multi-physics simulations in this manner, we have produced a 'computational microscope' that enables access to all of the voltages and currents throughout the entire structure, revealing behaviours that would have otherwise been hidden using conventional modeling approaches. The insights gained using these techniques will be very useful for future designs.

\section{ACKNOWLEDGMENTS}

We would like to acknowledge our Freescale colleagues Chris Dragon for designing the transistors studied in this paper, Eddie Mares for IR thermal measurements, and Jeff Crowder for stimulating thermal modeling discussions. We would also like to acknowledge the team at Sonnet Software Inc., for their assistance in the electromagnetic visualization of the manifold currents.

\section{REFERENCES}

[1] M. Rudolph and W. W. Heinrich, "Assessment of power-transistor package models: Distributed versus lumped approach," in European Microwave Integrated Circuits Conference (EuMIC), Sep. 2010, pp. 8689.

[2] T. Johansson and T. Arnborg, "A novel approach to 3-D modeling of packaged RF power transistors," IEEE Trans. Microw. Theory Tech., vol. 47, no. 6, pp. 760-768, Jun. 1999.

[3] T. Liang, J. Pla, P. H. Aaen, and M. Mahalingam, "Equivalent-circuit modeling and verification of metal-ceramic packages for RF and microwave power transistors," IEEE Trans. Microw. Theory Tech., vol. 47, no. 6, pp. 709-714, Jun. 1999.

[4] P. H. Aaen, J. A. Pla, and C. A. Balanis, "Modeling techniques suitable for CAD-based design of internal matching networks of high-power RF/microwave transistors," IEEE Trans. Microw. Theory Tech., vol. 54, no. 7, pp. 3052-3059, Jul. 2006.

[5] K. Mouthaan, "Modeling of RF high power bipolar transistors," $\mathrm{Ph} . \mathrm{D}$. dissertation, Delft University of Technology, Delft, Netherlands, 2001.

[6] D. Denis, C. M. Snowden, and I. C. Hunter, "Coupled electrothermal, electromagnetic, and physical modeling of microwave power FETs," IEEE Trans. Microw. Theory Tech., vol. 54, no. 6, pp. 2465-2470, Jun. 2006.

[7] K. Goverdhanam, W. Dai, M. Frei, D. Farrell, J. Bude, H. Safar, M. Mastrapasqua, and T. Bambridge, "Distributed effects in high power RF LDMOS transistors," in IEEE MTT-S Int. Microwave Symp. Dig., Jun. 2005, pp. 333-336.

[8] D. Resca, A. Santarelli, A. Raffo, R. Cignani, G. Vannini, F. Filicori, and D.-R. Schreurs, "Scalable nonlinear FET model based on a distributed parasitic network description," IEEE Trans. Microw. Theory Tech., vol. 56, no. 4, pp. 755-766, Apr. 2008.

[9] D. Resca, J. Lonac, R. Cignani, A. Raffo, A. Santarelli, G. Vannini, and F. Filicori, "Accurate EM-based modeling of cascode FETs," IEEE Trans. Microw. Theory Tech., vol. 58, no. 4, pp. 719-729, Apr. 2010.

[10] B. Breitkreutz, F. Schmuckle, and W. Heinrich, "Feeding structures for packaged multifinger power transistors," in European Microwave Conference, Oct. 2007, pp. 146-149.

[11] S. Lee, P. Roblin, and O. Lopez, "Modeling of distributed parasitics in power FETs," IEEE Trans. Electron Devices, vol. 49, no. 10, pp. 1799-1806, Oct. 2002. 


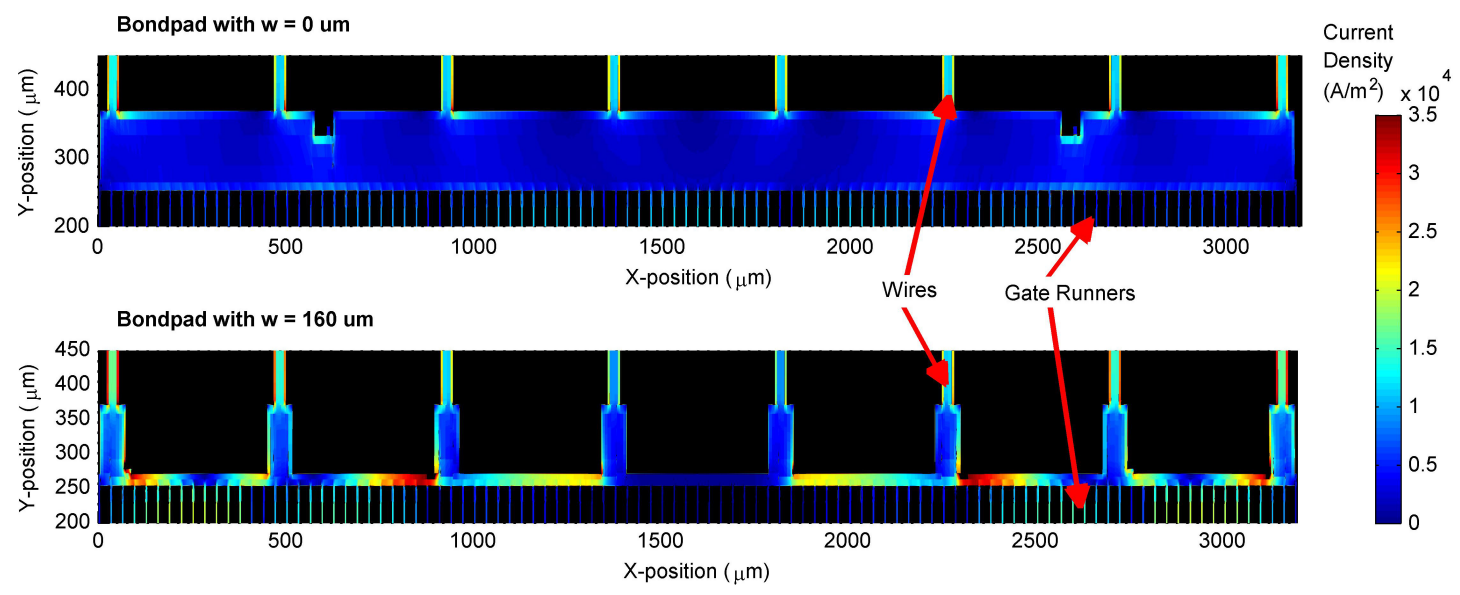

Fig. 19. Visualization of the fundamental component of current vs. position within the gate bondpads for the $w=0 \mu \mathrm{m}$ and $w=160 \mu \mathrm{m}$ cases.

[12] M. Rudolph, C. Fager, and D. E. Root, Nonliner Transistor Model Parameter Extraction Techniques. Cambridge, UK: Cambridge University Press, 2011, ch. 3, pp. 43-83.

[13] P. H. Aaen, J. Wood, D. Bridges, L. Zhang, E. Johnson, T. Barbieri, J. Pla, C. M. Snowden, J. P. Everett, and M. J. Kearney, "Multi-physics modeling of high-power microwave transistors," in IEEE MTT-S Int. Microwave Symp. Dig., Jun. 2012, pp. 1-3.

[14] P. H. Aaen, J. A. Plá, and J. Wood, Modeling and Characterization of RF and Microwave Power FETs. Cambridge, UK: Cambridge University Press, 2007.

[15] R. Grondin, S. El-Ghazaly, and S. Goodnick, "A review of global modeling of charge transport in semiconductors and full-wave electromagnetics," IEEE Trans. Microw. Theory Tech., vol. 47, no. 6, pp. 817-829, Jun. 1999

[16] A. Cidronali, G. Collodi, G. Vannini, and A. Santarelli, "A new approach to FET model scaling and mmic design based on electromagnetic analysis," IEEE Trans. Microw. Theory Tech., vol. 47, no. 6, pp. 900907, Jun. 1999.

[17] D. Guerra, D. K. Ferry, M. Saraniti, and S. M. Goodnick, "Millimeterwave power amplifier circuit-device simulations through coupled harmonic balance-monte carlo particle-based device simulator," in IEEE MTT-S Int. Microwave Symp. Dig., Jun. 2012.

[18] S. Luniya, W. Batty, V. Caccamesi, M. Garcia, C. Christoffersen, S. Melamed, W. Rhett Davis, and M. Steer, "Compact electrothermal modeling of an X-band mmic," in IEEE MTT-S Int. Microwave Symp. Dig., Jun. 2006, pp. 651-654.

[19] J. Wood, P. H. Aaen, D. Bridges, D. Lamey, M. Guyonnet, D. S. Chan, and N. Monsauret, "A nonlinear electro-thermal scalable model for highpower RF LDMOS transistors," IEEE Trans. Microw. Theory Tech., vol. 57, no. 2, pp. 282-292, Feb. 2009.

[20] J. Wood, D. Lamey, M. Guyonnet, D. Chan, D. Bridges, N. Monsauret, and P. H. Aaen, "An extrinsic component parameter extraction method for high power RF LDMOS transistors," in IEEE MTT-S Int. Microwave Symp. Dig., Jun. 2008, pp. 607-610.

[21] D. E. Root, "Charge modeling and conservation laws," in Asia-Pacific Microwave Conference Workshop WS2, Modeling and Characterization of Microwave Devices and Packages', Sydney, Australia, Jun. 1999.

[22] C. Fager, J. C. Pedro, N. B. de Carvalho, and H. Zirath, "Prediction of IMD in LDMOS transistor amplifiers using a new large-signal model," IEEE Trans. Microw. Theory Tech., vol. 50, no. 12, pp. 2834-2842, Dec. 2002.

[23] D. Bridges, J. Wood, M. Guyonnet, and P. H. Aaen, "A nonlinear electrothermal model for high power RF LDMOS transistors," in IEEE MTT-S Int. Microwave Symp. Dig., Jun. 2008, pp. 475-478.

[24] P. C. Canfield, S. C. F. Lam, and D. J. Allstot, "Modeling of frequency and temperature effects in GaAs MESFETs," IEEE J. Solid State Circuits, vol. 25, no. 1, pp. 299-306, Feb. 1990.

[25] P. H. Aaen, J. Wood, Q. Li, and E. Mares, "Thermal resistance modeling for the electrothermal layout of high-power RF transistors," in IEEE MTT-S Int. Microwave Symp. Dig., May 2010, pp. 1672-1675.
[26] J. Sofia, "Fundamentals of thermal resistance measurement," 1995, analysis Tech., Inc. [Online]. Available: www.analysistech.com/ downloads/fundamen.pdf

[27] P. H. Aaen, J. A. Pla, and C. A. Balanis, "On the development of CAD techniques suitable for the design of high-power RF transistors," IEEE Trans. Microw. Theory Tech., vol. 53, no. 10, pp. 3067-3074, Oct. 2005.

[28] J. F. Sevic, "A sub $1 \Omega$; load-pull quarter-wave pre-matching network based on a two-tier TRL calibration," in ARFTG Conference Digest-Fall, 52nd, Nov. 1998, pp. 73-81.

[29] P. H. Aaen, J. A. Pla, D. Bridges, and E. Shumate, "A wideband method for the rigorous low-impedance loadpull measurement of highpower transistors suitable for large-signal model validation," in ARFTG Conference Digest-Fall, 56th, Nov. 2000, pp. 1-7.

[30] J. Sirois and B. Noori, "Tuning range analysis of load pull measurement systems and impedance transforming networks," in ARFTG Conference Digest-Spring, 69th, Jun. 2007, pp. 1-5.

[31] Y. Gao, A. Lauer, Q. Ren, and I. Wolff, "Calibration of electric coaxial near-field probes and applications," IEEE Trans. Microw. Theory Tech., vol. 46, no. 11, pp. 1694-1703, Nov. 1998.

[32] S. Cripps and A. Porch, "An active, non-intrusive, high resolution microwave field probe with applications in high power RF device and circuit design," in Wireless and Microwave Technology Conference (WAMICON), 2010 IEEE 11th Annual, Apr. 2010, pp. 1-4.

[33] K. Yang, G. David, S. Robertson, J. Whitaker, and L. Katehi, "Electrooptic mapping of near-field distributions in integrated microwave circuits," IEEE Trans. Microw. Theory Tech., vol. 46, no. 12, pp. 2338-2343, Dec. 1998.

[34] M. Mahalingam and E. Mares, "Infrared temperature characterization of high power RF devices," in IEEE MTT-S Int. Microwave Symp. Dig., vol. 3, May 2001, pp. 2199-2202.

[35] P. H. Aaen, L. Zhang, D. Lamey, J. Wood, and S. Arvas, "Visualizing time-domain spatially distributed electromagnetic fields using Sonnet," in 28th International Reivew of Progress in Applied Computational Electromagnetics (ACES), Columbus, OH, Apr. 2012. 


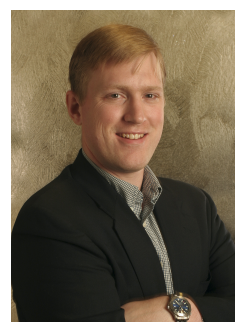

Peter H. Aaen (S'93, M'97, SM'07) received the B.A.Sc. degree in Engineering Science and the M.A.Sc. degree in Electrical Engineering, both from the University of Toronto, Toronto, ON., Canada, and the Ph.D. degree in Electrical Engineering from Arizona State University, Tempe, AZ., USA, in 1995,1997 and 2005, respectively. $\mathrm{He}$ is the Manager of the RF Modeling and Measurement Technology team of the RF Division of Freescale Semiconductor, Inc, Tempe, AZ, USA; a company which he joined in 1997 (then Motorola Inc. Semiconductor Product Sector). His current work focuses on the development and validation of multi-physics based modeling methodologies for highpower and high-frequency electronic devices. He co-authored Modeling and Characterization of RF and Microwave Power FETs (Cambridge University Press, 2007) and has authored over thirty papers, articles and workshops in the fields of electromagnetic simulation, package modeling, and microwave device modeling and characterization.

$\mathrm{He}$ is a member of the Microwave Theory and Techniques Society, a member of the ARFTG Executive Committee, and is an active member of many technical committees including: MTT-1 Computer-Aided Design and the technical program committee (TPC) of the IEEE Conference on Electrical Performance of Electronic Packaging and Systems.

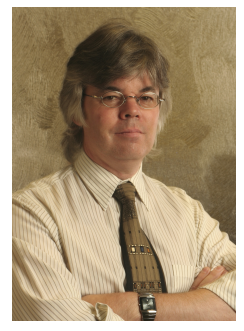

John Wood (M'87, SM'03, F'07) Received B.Sc and Ph.D. degrees in Electrical and Electronic Engineering from the University of Leeds, UK, in 1976 and 1980, respectively. He is currently a Senior Principal Member of the Technical Staff at Maxim Labs, Maxim Integrated Products, Inc, responsible for system simulation for RFICs. He was formerly a Distinguished Member of Technical Staff responsible for RF CAD \& Modeling in the RF Division of Freescale Semiconductor, Inc, Tempe, AZ, USA, where the work in this paper was carried out. From 1997-2005 he worked in the Microwave Technology Center of Agilent Technologies (then Hewlett Packard) in Santa Rosa, CA, USA. His areas of expertise include the development of compact device models and nonlinear behavioral models for RF power transistors and ICs. He is a Fellow of the IEEE, and a member of the Microwave Theory and Techniques, and Electron Devices Societies.

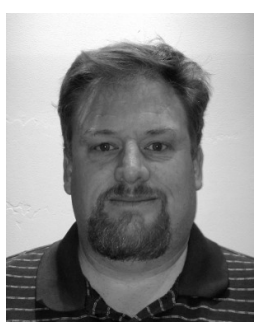

Daren Bridges (M'98) received the B.S. degree in electrical engineering from the University of Utah in 1992 and the M.S. degree in electrical engineering from the University of Texas at Dallas in 1996. In 1992, he joined the RF/Microwave Group at Texas Instruments and specialized in linear MESFET and HEMT modeling for MMIC devices. In 1997, he joined the Modeling team of the RF Division of Motorola Inc. Semiconductor Products Sector (Freescale Semiconductor Inc.) His areas of expertise include RF high power nonlinear model development and implementation within harmonic-balance simulators and the building and testing of active component model libraries. He is author or co-author on several papers, articles and workshops in the areas of RF and microwave device modeling and simulation.

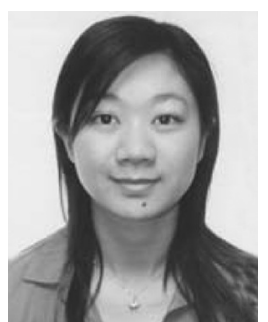

Lei Zhang (S'03,M'09) received the B.Eng. degree in electrical engineering (with a minor in economics) from Tianjin University, Tianjin, China, in 2000, and the M.A.Sc. and Ph.D. degrees in electrical engineering from Carleton University, Ottawa, ON, Canada, in 2003 and 2008, respectively. She is currently a Modeling Engineer with the RF Modeling Team, RF Division, Freescale Semiconductor Inc., Tempe, AZ. Her expertise includes space mapping, neural networks, automatic model generation of passive and active components, and application of computeraided design for RF circuits and systems. She has authored or coauthored over 20 technical papers and articles published in journals, conference proceedings, and international workshop notes.

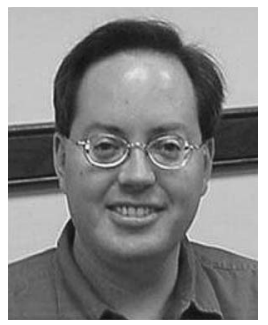

Eric Johnson (M'92) received the B.S. degree in electrical engineering from the University of Michigan, Ann Arbor, in 1989 and the M.S. degree in electrical engineering from the National Technological University, Fort Collins, CO, in 1994. In 1989, he joined Motorola (now Freescale Semiconductor Inc.), Tempe, AZ, working primarily in the area of RF and microwave device modeling and characterization for IIIV devices. Since 2007, he has been a member with the RF Division, Freescale Semiconductor Inc., where he has been responsible for RF LDMOS device modeling and characterization. He is the author or coauthor of a number of papers in the areas of RF and microwave modeling and measurements. His research interests include measurement techniques, measurement automation, and nonlinear device modeling.

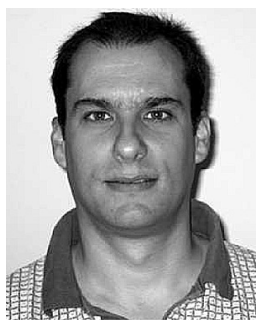

Jaime A. Plá (S'87, M'91) was born in Mayagez, PR, in 1969. He received the B.S. degree in electrical engineering from the University of Puerto Rico, Mayagez, in 1991, and the M.S. degree in microwave engineering from the University of Massachusetts at Amherst, in 1993. In 1991, he joined the Microwave Semiconductor Laboratory, Research Division, Raytheon, Lexington, MA, where he was primarily involved with the development of microwave measurement techniques and linear and nonlinear models for monolithic-microwave integrated-circuit (MMIC) semiconductor devices such as GaAs MESFETs pseudomorphic high electron-mobility transistors (pHEMTs), and HBTs. In 1995, he joined the Wireless Infrastructure Systems Division, Semiconductor Product Sector, Motorola (now Freescale Semiconductor Inc.), Phoenix, AZ, where his research is currently centered on the development of high-power RF electrothermal device models for LDMOS devices. His other areas of current interest are the development of package modeling techniques and modeling of passive components, as well as techniques for the measurement of electrical and thermal transistor characteristics related to small- and large-signal modeling extraction and validation.

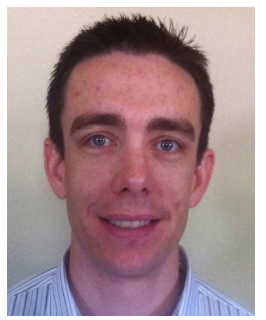

Travis Barbieri joined Freescale Semiconductor Inc. in 2005 as an RF Technician. He has provided technical support for several projects dealing with the development and modeling of high power LDMOS devices. His professional interests include expanding his knowledge of microwave measurements and modeling techniques. He is currently perusing a degree in electrical engineering at Arizona State University. 


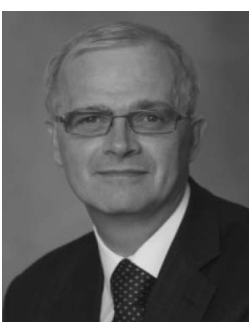

Christopher M. Snowden (S'82,M'82,SM'91,F'96) received the B.Sc. (Hons.), M.Sc., and Ph.D. degrees from the University of Leeds, Leeds, U.K. After graduating in 1977, he worked as an Applications Engineer for Mullard. His Ph.D. studies were later conducted in association with Racal-MESL. From 1992 to 2005, he held the personal Chair of microwave engineering with the University of Leeds. During the period of 1995-1998, he was the Head with the Department and, subsequently, Head with the School of Electronic and Electrical Engineering. He was the first Director of the Institute of Microwaves and Photonics, School of Electronic and Electrical Engineering. From 1989 to 1998, he was a Consultant with M/A-COM Inc. In 1998, he joined Filtronic plc as the Director of technology and, in 1999, was promoted to a Joint Chief Executive. Until April 2005, he was the Chief Executive of Filtronic ICS and a Professor of Microwave Engineering with the University of Leeds. He is currently the President and Vice-Chancellor of the University of Surrey, Surrey, U.K. He was Knighted by the Queen in the UK New Years Honours in 2012 for services to Engineering and Higher Education. He is the author of eight books, over 330 refereed journal and conference papers, and many other articles. $\mathrm{He}$ was the President of the Institution of Engineering and Technology in 200910 and is Vice-President of the Royal Academy of Engineering. His main research interests include semiconductor device modeling and microwave circuit technology and design. Sir Christopher was the recipient of the 1999 Microwave Prize and the 2009 Distinguished Educator Award of the IEEE Microwave Theory and Techniques Society. In 2004, he was awarded the Silver Medal of the Royal Academy of Engineering for his contributions to the compound semiconductor industry. He has received the 2012 Outstanding Career Award by the European Microwave Association, He is a Fellow of the Royal Society, the Royal Academy of Engineering, and the Institute of Engineering and Technology. He was a Distinguished Lecturer for the IEEE Electron Devices Society until 2005.

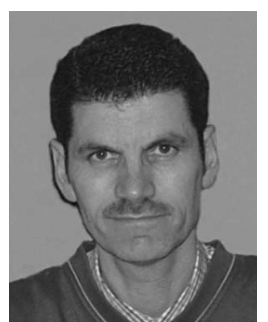

John P. Everett received the B.Sc.(Hons) degree in physics and astrophysics from Queen Mary and Westeld College, London, U.K., in 1994. In 1998, he received the Ph.D. degree in high-temperature superconductivity and the Diploma of Imperial College in experimental solid-state physics. After graduating, he continued his work with Imperial College as a Postdoctoral Researcher investigating direct- and alternating-current losses in high-temperature superconducting tapes. In 2006, he joined the Advanced Technology Institute (ATI), University of Surrey, Surrey, U.K., to work on modeling molecular systems with the support of the Daphne Jackson Trust. Since 2008, he has been with the ATI, developing nonlinear compact models for radio-frequency power laterally diffused metal oxide semiconductor devices.

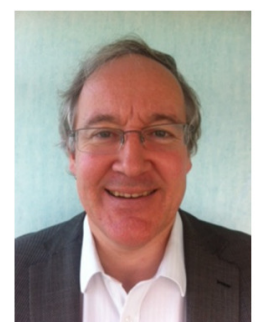

Michael J. Kearney received the B.A. degree in Physics from the University of Oxford in 1985, and the $\mathrm{PhD}$ degree from the University of Warwick in 1988. In 1988 he joined the Long Range Research Laboratory at the GEC Hirst Research Centre, London. After serving a period as Manager of that Laboratory, in 1995 he joined the Department of Electronic and Electrical Engineering at Loughborough University, serving as Head of Department between 1997 and 2000. He joined Surrey University in 2002 as the inaugural Director of the Advanced Technology Institute, and was appointed Head of the School of Electronics and Physical Sciences in 2005. In 2007 he assumed the role of Dean of the Faculty of Engineering and Physical Sciences, and in 2012 he became a Pro ViceChancellor of the University with responsibility for the Research Excellence Framework. The author of over 100 publications, his research interests include the modelling and simulation of devices for RF and microwave applications, SiGe FETs and thin-film solar cells. 